\title{
Expression and Branch-Specific Export of mRNA Are Regulated by Synapse Formation and Interaction with Specific Postsynaptic Targets
}

\author{
Samuel Schacher, ${ }^{1}$ Fang Wu, ${ }^{1}$ John D. Panyko, ${ }^{1}$ Zhong-Yi Sun, ${ }^{1}$ and Denong Wang ${ }^{2}$ \\ ${ }^{1}$ Center for Neurobiology and Behavior and ${ }^{2}$ Genome Center, Columbia University College of Physicians and Surgeons \\ and New York State Psychiatric Institute, New York, New York 10032
}

\begin{abstract}
Mechanosensory neurons (SNs) of Aplysia form synapses in culture with some targets (L7), but not others (L11), even when a $\mathrm{SN}$ is plated with both targets. We examined whether branchspecific net export of mRNA encoding synapse-specific molecules might contribute to branch-specific synapse formation. Single-cell RT-PCR was used to assay levels of mRNA encoding the SN-specific neuropeptide (sensorin A) and other transcripts in cell bodies and neuritic processes of SNs cultured alone or with synaptic targets. Some mRNAs are exported to neurites, but not others. Sensorin A mRNA is detected only in $\mathrm{SN}$ cell bodies and neurites, and expression levels correlate with the strength of the synaptic connections formed with L7 after $4 \mathrm{~d}$ in culture. After $4 \mathrm{~d}$, more sensorin A transcripts are detected in SN neurites contacting L7 than in SN neurites
\end{abstract}

The correct wiring of the CNS is believed to result from a multistep process involving axon guidance, target selection, and fine tuning of connectivity via activity-dependent mechanisms. In some circumstances individual neurons undergo branch-specific synapse formation and elimination simultaneously. Local signals, diffusible or cell surface molecules, may modulate synapse formation or elimination (for review, see Cabelli et al., 1997; Fitzsimonds and Poo, 1998; Sanes et al., 1998; Winberg et al., 1998). How do these signals mediate the long-term maintenance of one set of synaptic connections and the decline in efficacy or elimination of others in the same neuron? One potential mechanism is that local signals modulate the synthesis and targeting of macrocmolecules required for synapse formation or maintenance. Although some intrinsic signals could instruct region-specific segregation-nucleus versus cytoplasm, basal versus apical surfaces of various cells, or axons versus dendrites of neurons-it is unclear how macromolecules might be segregated or targeted to specific regions within a given structure.

Signals that modulate mRNA export or local translation could target macromolecules to specific sites within pre- and/or postsynaptic elements of neurons (Martin et al., 1997; Steward et al.,

Received Dec. 23, 1998; revised May 17, 1999; accepted May 17, 1999.

This research was supported by National Institutes of Health (NIH) Grant NS 27541 and National Science Foundation Grant IBN-9808938; animals were provided by the National Center for Research Resources National Resource for Aplysia at the University of Miami, supported by NIH Grant RR-10294. We thank Rachel Yarmolinsky for assistance in preparing the figures and Drs. A. Hegde, R. Hen, and Z.-P. Sun for comments on this manuscript.

Correspondence should be addressed to Dr. Samuel Schacher, Center for Neurobiology and Behavior, Columbia University College of Physicians and Surgeons, New York State Psychiatric Institute, 722 West 168th Street, New York, NY 10032. Copyright (C) 1999 Society for Neuroscience $0270-6474 / 99 / 196338-10 \$ 05.00 / 0$ contacting L11. The differential expression at $4 \mathrm{~d}$ is found even when a single SN contacts both targets simultaneously. By contrast, no significant difference in expression is detected in SN neurites contacting L7 versus L11 after $1 \mathrm{~d}$ of coculture. The results suggest that interaction and synapse formation with a specific target lead to a time-dependent change in the branchspecific accumulation of sensorin A mRNA in SNs. Because local protein synthesis at synaptic sites might contribute to synaptic function or plasticity, the results suggest that branchspecific targeting of mRNA encoding synapse-related molecules may contribute to the formation of specific synapses.

Key words: synapse formation; specificity; gene expression; mRNA export; RT-PCR; cell culture; Aplysia

1998; Wu et al., 1998). The export of specific mRNAs into dendrites of vertebrate neurons is well established (Crino and Eberwine, 1996; Knowles et al., 1996; Steward, 1997). Axons of vertebrate neurons, during regeneration in culture (Kleiman et al., 1994; Olink and Hollenbeck, 1996; Bassell et al., 1998) and in the posterior pituitary (Mohr et al., 1991), also contain specific mRNAs. Some mRNAs, including those encoding neuropeptides and other proteins, are exported into axons of invertebrate neurons (van Minnen, 1994). Isolated axons of invertebrate neurons appear to have the machinery to synthesize proteins (van Minnen et al., 1997), including the proteins required for new synapse formation accompanying long-term synaptic plasticity (Martin et al., 1997). Thus, local translation of exported mRNAs encoding synapse-specific macromolecules may contribute to the formation or maintenance of specific synapses.

The large identified neurons of Aplysia form specific synapses in dissociated cell culture even when a presynaptic neuron is confronted with multiple targets (Camardo et al., 1983; Schacher and Montarolo, 1991; Hawver and Schacher, 1993; Casadio et al., 1997). Contact with a synaptic target by a mechanosensory neuron (SN) leads to expression of branch-specific morphological, physiological, and biochemical features. These include the formation of varicosities with active zones (Glanzman et al., 1989), expression of electrical excitability and synaptic plasticity (Sun and Schacher, 1996), and expression of high levels of the neuropeptide sensorin A (Santarelli et al., 1996; Casadio et al., 1997). The level of sensorin A peptide in the SN branches contacting L7 correlates with changes in synaptic efficacy associated with new synapse formation, long-term facilitation with 5-HT, and longterm depression with FMRFamide.

Can branch-specific export of mRNAs contribute to branch- 
specific synapse formation? To test this hypothesis, we combined single-cell RT-PCR with the advantages of cell cultures consisting of individual pre- and postsynaptic neurons plated either alone or in various combinations. We confirmed that sensorin mRNA is expressed only in SNs and found that some mRNAs, actin and sensorin A, are exported from the cell body. Accumulation of sensorin A mRNA to SN terminals is regulated by contact with an appropriate target (L7) with time in culture even when branches of the same SN contact another target (L11) that fails to induce synapse formation. The results support the idea that branchspecific export or accumulation of mRNAs encoding synapsespecific molecules may contribute to the formation or maintenance of specific synapses.

\section{MATERIALS AND METHODS}

Cell culture and electrophysiology. Mechanosensory neurons (SNs) were isolated from pleural ganglia dissected from adult animals (80-100 gm); motor cell L7 and/or L11 were isolated from juvenile (1-3 gm) abdominal ganglia and maintained in culture for $20 \mathrm{hr}$ or $4 \mathrm{~d}$ as described previously (Schacher, 1985; Rayport and Schacher, 1986; Schacher and Montarolo, 1991; Z hu et al., 1994). Individual cells (SN, L7, and/or L11) were plated in the same culture dish at very low density so that the cells did not contact each other (Fig. 1A). Cocultures consisted of one SN with one L7 or one L11 (SN-L7 or SN-L11; Fig. 1B), one SN with two L7 (SN-L7/L7), and one SN with one L7 and one L11 (SN-L7/L11; Fig. 1C) (see Martin et al., 1997). Because each neuron was isolated from the rest of the nervous system (Schacher, 1985), the glial cells typically did not contaminate the cultures. Occasionally, a few glial cells were attached to the cell body of L7 or L11. No glial cells were detected in the compartment containing the distal axon and regenerated neurites.

Standard electrophysiological techniques were used to record the amplitude of the EPSP evoked in L7 or L11 with the stimulation of each SN (Schacher and Montarolo, 1991; Sun and Schacher, 1996; Martin et al., 1997). The motor cells (L7 and L11) were impaled with a microelectrode (resistance of 15-20 M $\Omega$ ) containing $2.0 \mathrm{M} \mathrm{K}$-acetate, $0.5 \mathrm{M} \mathrm{KCl}$, and 10 mM K-HEPES, pH 7.4, and held at $-80 \mathrm{mV}$. Each SN was stimulated with a brief $(0.3-0.5 \mathrm{msec})$ depolarizing pulse to evoke an action potential, using an extracellular electrode placed near the cell body of the SN. The amplitude of the EPSPs evoked in $\mathrm{L} 7$ ranged from $1 \mathrm{mV}$ (day 1) to $42 \mathrm{mV}$ (day 4). Action potentials in SNs never evoked EPSPs $(<0.2$ $\mathrm{mV}$ ) in L11 (data not shown) (see Glanzman et al., 1989; Schacher and Montarolo, 1991).

Dissection of cellular compartments. Cultures were rinsed with hemolymph-free medium (1:1 by volume of L15 plus Instant Ocean) and allowed to cool to $4^{\circ} \mathrm{C}$ for $\sim 20 \mathrm{~min}$. Bovine serum albumin (BSA; 10 $\mu \mathrm{g} / \mathrm{ml}$ ) was added to the final rinse to prevent dissected cell bodies and neurites from sticking to the dish surface or the transfer pipettes. Cellular compartments were dissected and transferred by using the methods described previously (Ambron et al., 1985). For cells plated alone in culture (Fig. $1 \mathrm{~A}$ ), we analyzed expression in two compartments: (1) cell body plus $100-150 \mu \mathrm{m}$ of proximal axon and attached neurites (CB) and (2) distal axon plus regenerated neurites emerging from axon stumps $(\mathrm{N})$. In some instances we combined the neuritic compartment $(\mathrm{N})$ of an isolated $\mathrm{SN}$ with the cell body and neuritic compartments $(\mathrm{CB}+\mathrm{N})$ of an isolated target neuron. In cocultures of one SN and one target (L7 or L11) we analyzed the expression in three compartments; cell body and proximal axon $(100-150 \mu \mathrm{m})$ of $\mathrm{SN}(1)$, cell body and proximal axon $(100-150 \mu \mathrm{m})$ of the target (2), and interacting distal axons and neurites from both cells ( 3 ; as shown in Fig. $1 B$ ). In some instances we combined compartments 2 and 3 to measure the total export of sensorin A transcripts in SN neurites contacting the entire target. For cultures with one $\mathrm{SN}$ in contact with two targets (two L7s or one L7 plus one L11) we dissected and analyzed three compartments: SN cell body and proximal axons (100-150 $\mu \mathrm{m}$ beyond bifurcation; see compartment 1 in Fig. $1 C$ ), $\mathrm{SN}$ neurites interacting with one target (see compartment 2 in Fig. 1C), and the other target (see compartment 3 in Fig. 1C).

$R T$-PCR to detect expression of specific $m R N A$ s. Each cell compartment was transferred to $200 \mu \mathrm{l}$ of Trizol (Life Technologies, Gaithersburg, $\mathrm{MD}$ ), and total RNA was isolated after chloroform extraction and isopropanol precipitation. cDNA from each sample was synthesized by using random hexamers as primers and reverse transcriptase (Superscript II). Aliquots $(2 \mu \mathrm{l})$ from each sample were used to amplify specific

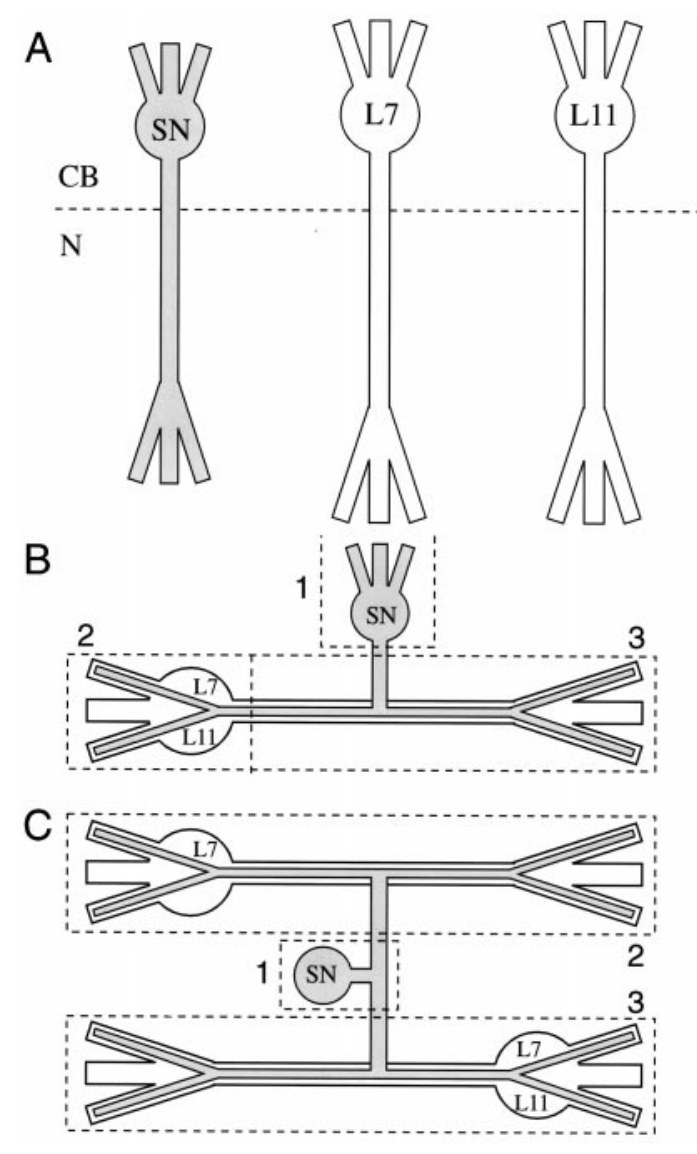

Figure 1. Compartments analyzed by RT-PCR. A, Isolated cells maintained in culture. Two compartments were analyzed: (1) Cell body and most proximal portion of axon and neurites $(C B)$ and (2) axon and regenerated neurites from axon stump $(N)$. In some experiments the neurite compartment of a single SN was added to both compartments of a single target cell cultured alone. $B$, Cocultures with SN contacting L7 or L11. In some experiments three compartments were analyzed: SN cell body (1), SN neurites contacting cell body and proximal axon of the target (2), and interacting neurites from both cells (3). In other experiments compartments 2 and 3 were combined as one. $C$, Cocultures with a SN contacting two targets (L7 and/or L11). Three compartments were analyzed: SN cell body (1); SN neurites contacting axon, neurites, and cell body of a L7 (2); and SN neurites contacting another L7 or L11 (3).

fragments by PCR (40 cycles), using specific primer sets for (1) SN-specific neuropeptide sensorin A (Brunet et al., 1991), AACAGAAACAGTCTTTCCCC and TCTTGACTCACCAACTGCC (nucleotides 43-331); (2) neuron-specific isoform of actin (DesGroseillers et al., 1994), CAGAGAGAAGATGACCCAG and GGGTAAGAGAAGCAAGAAAG (nucleotides 416-1298); (3) common extracellular region for all isoforms of apCAM (Mayford et al., 1992), AACAACACGAAGATCGAAG and TGTTCACAATGCCATCAG (nucleotides 528-1104); and (4) molluscan 18S rRNA (Winnepenninckx et al., 1998), AAAACCAATCGTCGTCTC and TTTTCGTCACTACCTCCC (nucleotides 197-477). The lengths of the synthesized fragments detected with ethidium bromide staining on $1.2 \%$ agarose gels were identical to those predicted from the known sequences in the database. For semiquantitative comparison of mRNA expression, the number of PCR cycles was adjusted initially to ensure linearity of the fluorescent signals of the fragments amplified by PCR (see Fig. 2B). Each PCR run included amplifications and the detection of specific fragments from known cDNAs of known concentrations (positive controls), amplifications of each fragment from samples of medium collected near cells after removal of the cells from the culture dish, and other negative controls.

Samples used for analysis had to meet the following criteria: (1) no contamination of genomic DNA occurred in the initial RNA preparation with PCR amplification of the RNA sample itself, (2) actin and rRNA 


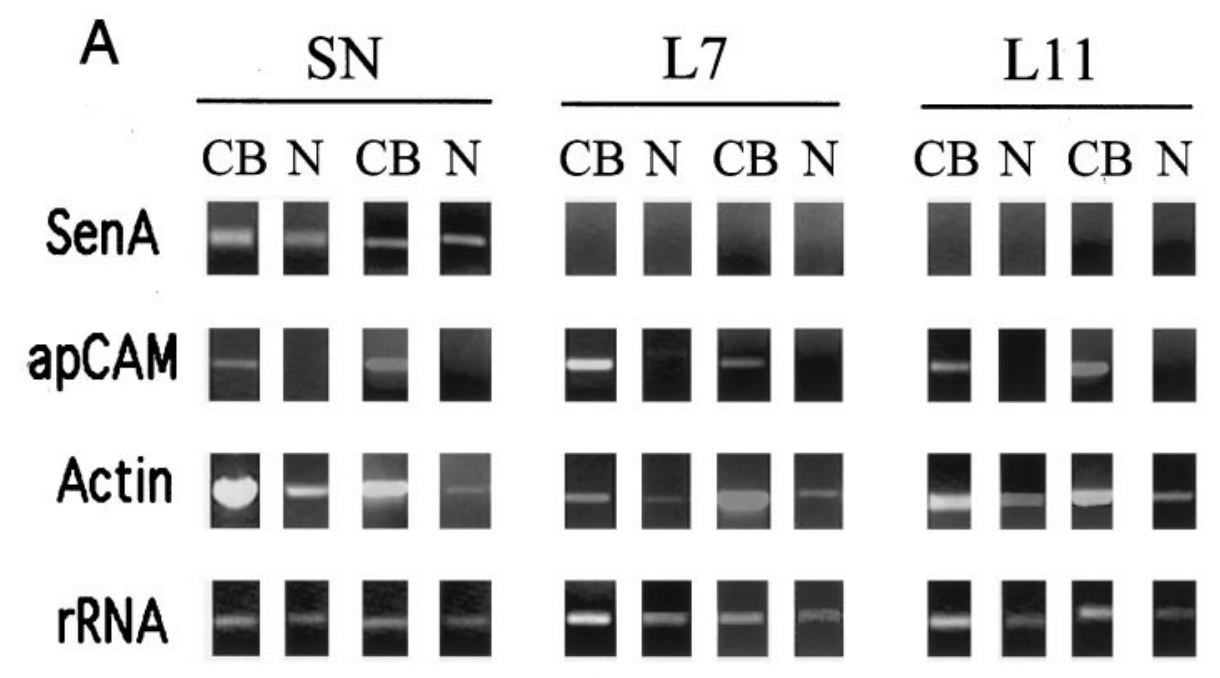

Figure 2. Detection of RNA in cell compartments by RT-PCR. $A$, Selective transcripts are exported, and sensorin A transcripts are expressed only in SNs. Shown is ethidium bromide staining of PCR fragments separated on $1.2 \%$ agarose gel. Fragments of appropriate lengths were amplified after 40 cycles with primer sets for sensorin A, 18S rRNA, actin, and apCAM by using two separate sets of samples from 4-d-old cultures of $S N, L 7$, and $L 11$ plated alone. Each cell was separated into two compartments, cell body $(C B)$ or neurites $(N)$. Actin and rRNA were detected in both compartments, whereas isoforms of apCAMs are detected only in the CB compartment. Sensorin A transcripts were detected only in the SNs and expressed in both CB and neurite compartments. $B$, PCR amplification is linear. Staining intensity of PCR fragments decreases with decreasing concentrations of cDNA in the sample. A 50-fold difference in cDNA levels (undiluted sample equals 100, and negative control is 0 ) can be resolved for the three mRNAs and the rRNA. If we normalize the highest concentration (100) as 1, the staining levels for the other concentrations (in descending order) are as follows: $0.58,0.24$, and 0.12 for sensorin A $(n=3) ; 0.52,0.32$, and 0.15 for apCAM $(n=3)$; $0.54,0.28$, and 0.16 for actin $(n=3) ; 0.49,0.32$, and 0.18 for rRNA $(n=3)$.

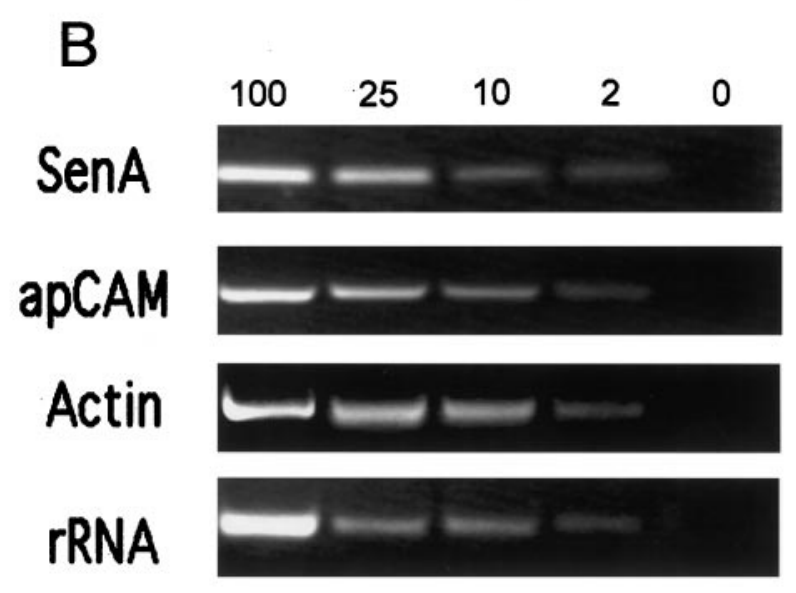

transcripts were detected within the linear range of the PCR amplification in each compartment of the culture or coculture, and (3) no fragments were detected after amplification of the cell culture medium collected after dissections. To quantify differences in the levels of sensorin A expression, we normalized staining intensity for sensorin A fragments $(\%)$ by the signals obtained for actin fragments in the same sample (staining intensity of sensorin A/staining intensity of actin $\times 100)$. We normalized sensorin A levels with actin levels, because average staining intensity for actin fragment within each compartment was not affected by cell interaction. METAMORPH software package was used to quantify average pixel intensities for PCR fragments in each compartment. Based on signals generated from known concentrations of sensorin A cDNA (positive controls), a twofold increase in staining intensity for sensorin A fragment equaled approximately a fivefold increase in the starting cDNA levels (see Fig. $2 B$ ). Overall differences in expression were calculated by ANOVAs ( 1 or 2 factors), and the significance of differences between individual groups was measured with a multicomparison test (Scheffé's $F$ test).

\section{RESULTS}

After $4 \mathrm{~d}$ in culture the large Aplysia neurons regenerate profusely (Schacher and Proshansky, 1983) and establish stable synaptic connections (Montarolo et al., 1986; Rayport and Schacher, 1986; Hawver and Schacher, 1993). Martin et al. (1997) found that $\mathrm{SN}$ axons and regenerated neurites isolated from their cell bodies synthesize significant amounts of proteins (10-20\% of total synthesis). We therefore examined whether the axons and neurites of single SN, L7, or L11 contain various transcripts. We measured levels of $18 \mathrm{~S}$ rRNA to confirm that all compartments of identified neurons have the capacity to translate mRNAs. We determined levels of actin mRNA, because these transcripts are abundant and are exported from the cell bodies of other neurons. We examined whether mRNA encoding a family of membrane proteins (apCAMs) is transported. This family of proteins, homologous to NCAM and fasciclin II, is the major membrane protein in neurons, accounting for $>10 \%$ of the total membrane protein in the CNS of Aplysia (Keller and Schacher, 1990; Mayford et al., 1992). We also determined whether the expression of a mRNA encoding a potential secretory product, the SN-specific neuropeptide sensorin A (Brunet et al., 1991), is regulated by interactions with specific targets. Levels of sensorin A peptide detected in SN varicosities are modulated with synapse formation and after treatments that evoke long-term changes in synaptic efficacy (Santarelli et al., 1996; Casadio et al., 1997).

\section{Specific transcripts are exported from cell bodies of Aplysia neurons}

Not all transcripts are exported from the cell bodies of Aplysia neurons. All cells contain $18 \mathrm{~S}$ rRNA in both cell body and neurites (Fig. 2A). The level of expression of the rRNA fragment in the cell bodies reflects differences in cell volume. The staining intensity of rRNA fragments in the cell bodies of L7 and L11 is approximately equal, and the levels are approximately two times greater than levels detected in the cell bodies of SNs $(n=5$ for 
each cell grown alone). A twofold change in staining intensity equals approximately a fivefold change in cDNA levels in the sample (Fig. $2 B$ ). The axons and neurites from individual identified cells also contained transcripts for some mRNAs (Fig. $2 A$ ). Actin transcripts were detected in the neuritic compartment of all of the cells that were examined. By contrast, apCAM transcripts were detected only in the cell bodies of SN, L7, and L11. As expected, transcripts for sensorin A were detected in SNs only and also were exported to SN axons and neurites.

\section{Expression and export of sensorin A mRNA are correlated with presence of synapses on day 4}

We next explored whether the expression of the SN-specific transcript is influenced by interaction with specific targets. Cocultures ( $\mathrm{SN}-\mathrm{L} 7$ and $\mathrm{SN}-\mathrm{L} 11)$ were prepared by placing the axon stump of a SN close to the axons of the targets, were allowed to regenerate for $4 \mathrm{~d}$ to form stable connections (Fig. 3), and then were examined for the presence of synaptic connections. As expected, all SN-L11 cultures had no synaptic connections, and all SN-L7 cultures formed connections. The amplitude of the EPSPs ranged from 3 to $>40 \mathrm{mV}$. In one set of cultures we assayed the expression of rRNA and mRNAs in three compartments (Figs. 1B, 3). In other cultures we assayed the expression of sensorin A and actin transcripts in two compartments: SN cell body and SN neurites contacting cell body and processes of the target cell (see compartments 2 and 3 in Fig. $1 B$ ). We also measured the expression of sensorin $\mathrm{A}$ and actin transcripts in the cell body and neurites of SNs cultured alone.

The expression of sensorin A transcripts in SNs correlated with the amplitude of the EPSP evoked in L7 (Fig. 4). As EPSP amplitude increased, there was a corresponding increase in the staining intensity of the PCR fragment for sensorin A in both the SN cell body and neuritic compartments (Fig. 4A, $B$, respectively). Staining intensity for actin fragments in the SN cell body did not correlate with EPSP amplitude $\left(R^{2}=0.08 ; p>0.3\right)$. There was a correlation between EPSP amplitude and staining intensity for sensorin A PCR fragments in the SN cell body $\left(R^{2}=0.35 ; p<\right.$ 0.02; Fig. $4 C$ ) and a stronger correlation between EPSP amplitude and staining intensity for sensorin A PCR fragments in SN neurites contacting L7 $\left(R^{2}=0.79 ; p<0.001\right.$; Fig. 4D). By contrast, staining intensity for actin fragments in the same samples containing combined neurites of SN and L7 did not correlate with EPSP amplitude $\left(R^{2}=0.01 ; p>0.8\right)$.

We next compared expression of sensorin A transcripts in SNs contacting L7 to expression in SNs contacting L11 (Fig. 5). As expected, rRNA and actin transcripts were present in all compartments, whereas apCAM transcripts were present in cell bodies only (Fig. $5 A$ ). The compartments with $\mathrm{SN}$ neurites interacting with cell body or processes of L7 contained sensorin A mRNA. By contrast, few sensorin A PCR fragments were detected in SN neurites interacting with cell body and processes of L11 (Fig. 5A). All SN cell bodies contained sensorin A transcripts. There was no significant effect of target interaction on the average level of sensorin A transcripts in SN cell bodies (ANOVA; $F=0.278 ; p>$ 0.75; Fig. $5 B$ ). On average, staining intensity for sensorin A PCR fragments within SN cell bodies ranged from 40 to $45 \%$ of the staining intensity detected for actin PCR fragments. By contrast, there was significantly greater staining for sensorin A fragments in SN neurites contacting L7 (Scheffé's $F=3.241 ; p<0.05$ ) and significantly less staining of sensorin A fragments in SN neurites contacting L11 (Scheffé's $F=2.981 ; p<0.05$ ) as compared with that detected in neurites of SNs plated alone (Fig. $5 B$ ). Overall,
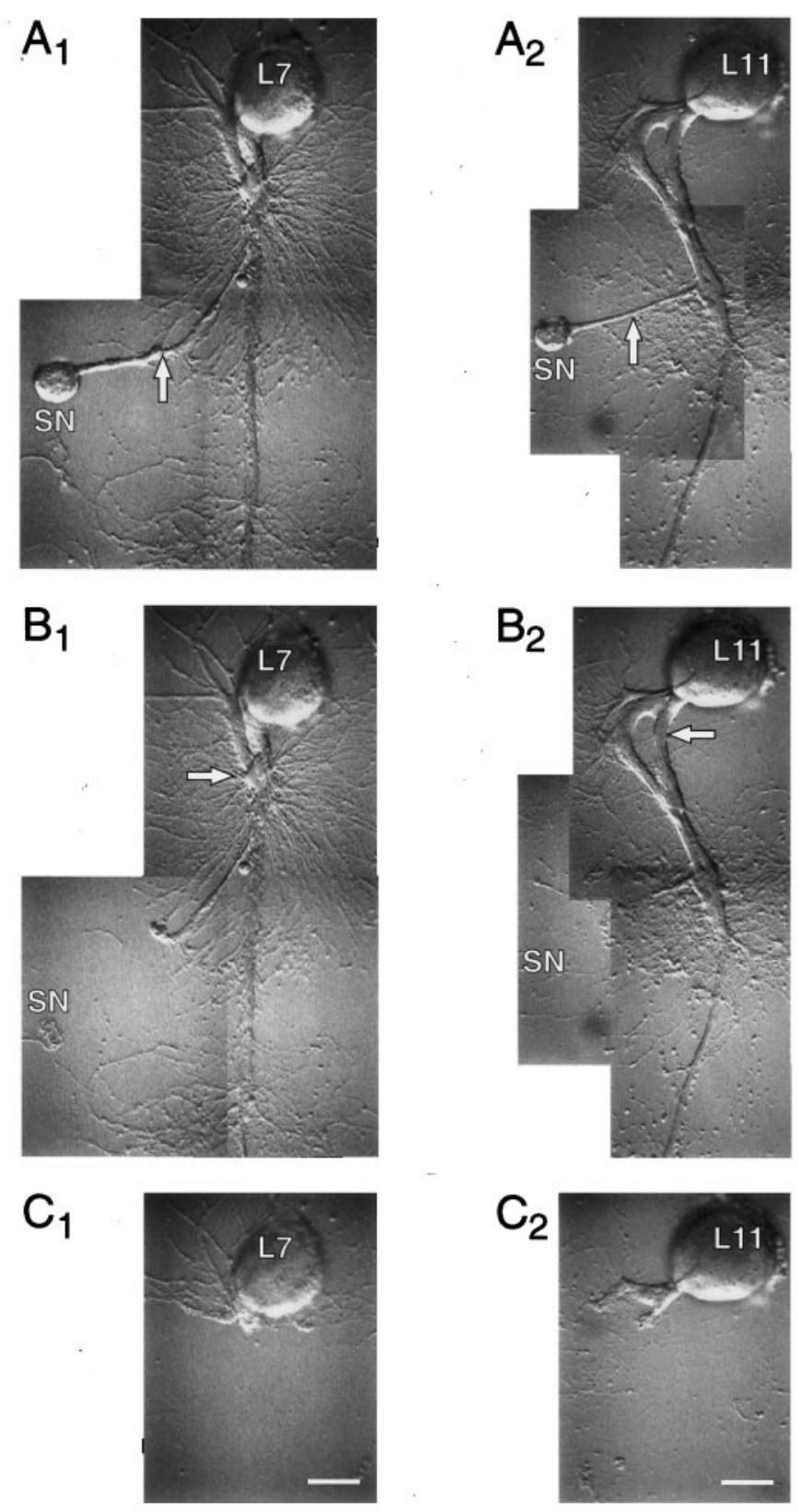

Figure 3. Dissection of cellular compartments for RT-PCR analyses. Shown are Nomarski contrast images of coculture before each dissection. Cell bodies or neuritic processes are detached from the substrate and transferred to tubes with Trizol. $A, \mathrm{SN}-\mathrm{L} 7\left(A_{1}\right)$ and SN-L11 $\left(A_{2}\right)$ cultures after $4 \mathrm{~d}$ before SN axons are cut (arrows). B, Same SN-L7 $\left(B_{1}\right)$ and $\mathrm{SN}-\mathrm{L} 11\left(B_{2}\right)$ cultures before target cell axon is cut (arrows). $C$, Remaining cell bodies and neurites before their detachment and transfer into Trizol. Each compartment is analyzed by RT-PCR. Scale bar, $60 \mu \mathrm{m}$.

absolute staining intensity for sensorin A PCR fragments in SN neurites contacting L7 was more than twofold greater than staining intensity in neurites of SNs plated alone (pixel intensity of $85.6 \pm 15.2$ vs $40.6 \pm 7.9$ ). Staining intensity in $\mathrm{SN}$ neurites contacting L11 was $<50 \%$ of the staining levels detected in SNs neurites plated alone $(18.3 \pm 4.9$ vs $40.6 \pm 7.9)$. Thus, target interaction can either enhance or depress net export of sensorin A transcripts to $\mathrm{SN}$ neurites and varicosities. Moreover, net export of sensorin A transcripts correlates with synaptic efficacy. 
A EPSP Amplitude ( $\mathrm{mV}$ )
B SN Neurites EPSP Amplitude (mV)

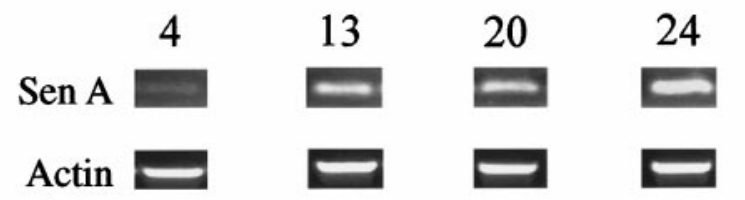

D
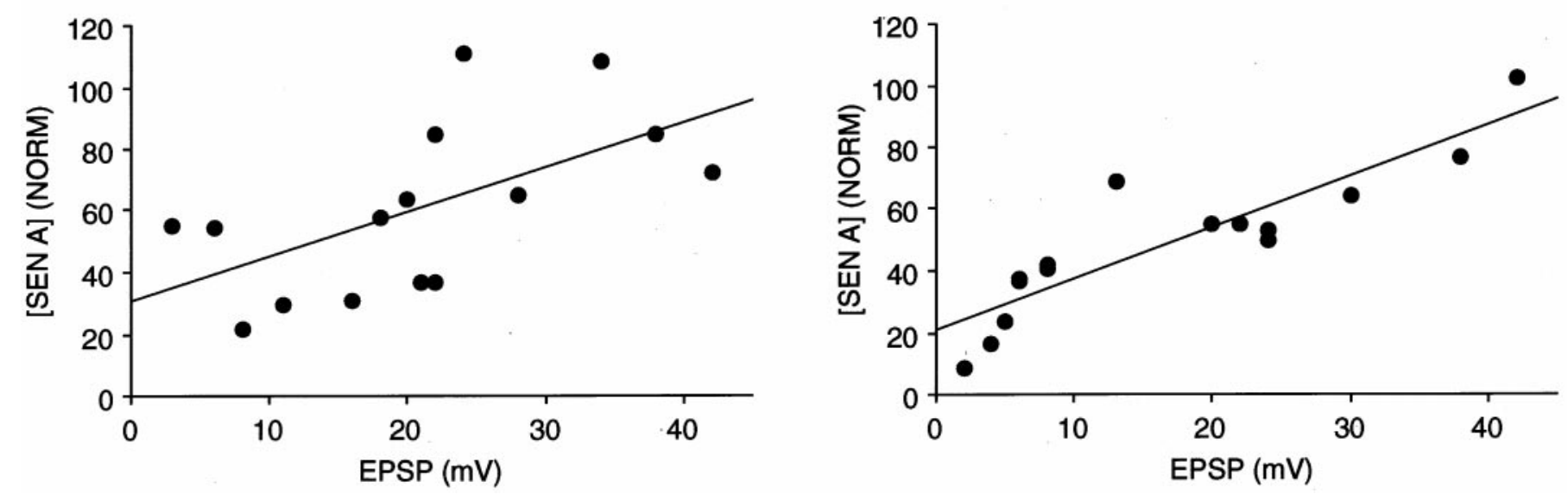

Figure 4. Expression of sensorin A transcripts in SNs correlates with synaptic efficacy. $A, C$, EPSP amplitude correlates with the expression of sensorin transcripts in SN cell bodies. Staining intensity of actin fragments amplified from SN cell bodies is not correlated with EPSP amplitude. Normalized levels of sensorin A fragment $(y$-axis = staining intensity of sensorin A/staining intensity of actin $\times 100)$ in the SN cell body (compartment 1 in Fig. $1 B$ ) are correlated with EPSP amplitude $\left(R^{2}=0.35 ; n=15 ; p<0.02\right)$. $B, D$, EPSP amplitude correlates with expression of sensorin A transcripts in SN neurites. Staining intensity of actin fragments in the neuritic compartments does not correlate with EPSP amplitude. Normalized levels of sensorin A fragment amplified in the SN neurites interacting with axons and neurites of L7 (compartment 3 in Fig. $1 B)$ correlate with EPSP amplitude $\left(R^{2}=0.79 ; n=15\right.$; $p<0.001)$.

\section{Branch-specific expression of sensorin A transcripts is regulated by targets on day 4}

We next examined whether net export of sensorin A mRNA is regulated in different branches of a single SN. We isolated SNs with bifurcate axons and plated a target neuron next to the distal stump of each branch. In some cultures we plated a L7 at each SN branch (Fig. 6A), whereas at other cultures we plated a L7 at one branch and a L11 at the other (Fig. 6B). As expected, stimulation of the SN evoked EPSPs in L7, but not in L11. Each culture was dissected into three compartments: $\mathrm{SN}$ cell body and proximal axons, SN neurites interacting with one target, and SN neurites interacting with the other (see Fig. $1 C$ ).

Overall expression of sensorin A transcripts in cell bodies of SNs was not affected significantly by the presence of two L7 targets versus one L7 and one L11 (SN lanes in Fig. $7 A ; p>0.5$ ). A significant difference was observed in the levels of sensorin A transcripts detected in the neurites of different $\mathrm{SN}$ branches $(n=$ 7 cultures; Fig. $7 B$ ). Whereas all $\mathrm{SN}$ neurites contacting $\mathrm{L} 7$ expressed sensorin A transcripts (L7 lanes in Fig. 7A), little or no expression of sensorin A transcripts was detected in SN neurites contacting L11 (L11 lanes in Fig. 7A). There was approximately a fourfold difference in the staining intensity of sensorin A PCR fragments in SN neurites contacting L7 versus neurites of the same SN contacting L11 (Scheffé's $F=3.869 ; p<0.04$ ). By contrast, equivalent staining of sensorin A PCR fragments was detected in the SN neurites contacting each $\mathrm{L} 7$ target (Fig. 7A, $C$; $n=7$ cultures; $p>0.7$ ).

\section{Target- and branch-specific differences in mRNA levels develop over time}

Under the culture conditions used, SNs establish synaptic connections with L7 by 12 hr (Zhu et al., 1994). By 20 hr, SN neurites extend over the major processes of L7 and form numerous varicosities (Zhu et al., 1994), and the new synaptic connections express some forms of short-term synaptic plasticity (Sun and Schacher, 1996). The efficacy of these connections increases with time in culture and reaches a stable level by $4 \mathrm{~d}$ (Glanzman et al., 1989; Zhu et al., 1994). We examined whether target- or branchspecific differences in the levels of sensorin A transcripts were expressed at these earlier stages of synapse formation (20 hr). We assayed for sensorin A transcripts in the neurites of SNs contacting L7 or L11 in cocultures with a single target (Fig. 8) and in cocultures in which a single SN contacts both targets (Fig. 9).

After $20 \mathrm{hr}$ the stimulation of the SN evoked EPSPs in L7 ranging from 1 to $16 \mathrm{mV}$. No responses were evoked in L11. There was no significant target-dependent difference in the level of actin or sensorin A transcripts in the cell bodies of SNs (Fig. 8; $p>0.6$ and 0.5 , respectively). Although average expression of sensorin A transcripts in SN neurites contacting L7 ( $n=6$ cultures) was greater than the level in SN neurites contacting L11 $(n=6$ cultures; Fig. $8 B$ ), we failed to detect a significant difference $(p>0.4)$ in the level of sensorin A transcripts in SN neurites contacting L7 versus L11. There was no significant difference $(p>0.3)$ in the level of sensorin A transcripts in branches contacting L7 versus L11 when SN contacted both 


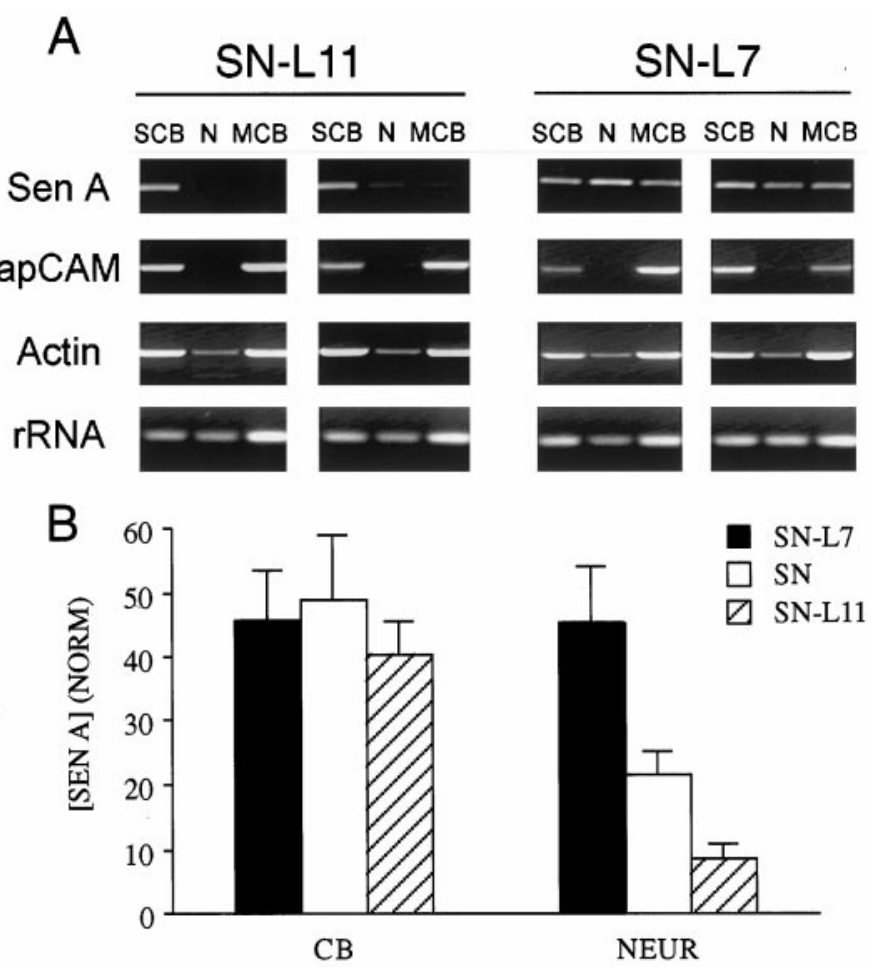

Figure 5. Export of sensorin A transcripts is modulated by target in $4 \mathrm{~d}$ cultures. $A$, RT-PCR fragments amplified from samples prepared from the cell bodies of SNs $(S C B)$, SN neurites interacting with axons and neurites of the target $(N)$, and SN neurites interacting with the cell body and proximal axon of the target motor cells $(M C B)$ from two sets of SN-L11 and SN-L7 cocultures. Fragments were synthesized with specific primers for sensorin A, actin, apCAM (all isoforms), and 18S rRNA. Note that sensorin A and actin mRNA were exported to neurites whereas apCAM was not. Little sensorin A was detected in the neurites of SN contacting the neuritic arbor or cell body of L11, compared with the signal obtained in the neurites of SN contacting the neuritic arbor and cell body of L7. B, Target-dependent modulation of levels of sensorin transcripts in SN neurites (normalized to the level of actin transcripts in each compartment). Two compartments were analyzed: SN cell body $(C B)$ and neurites of SN interacting with the entire target cell (NEUR). The neurite compartment of SN alone consisted of the neurites of a single SN combined with the cell body and neuritic arbor of a single target cell grown alone. Interaction with a target ( $n=7$ cultures for each condition) had no significant effect on the normalized expression of sensorin A transcripts in the SN cell bodies (see Results). By contrast, the expression of sensorin transcripts in the $\mathrm{SN}$ neurites was modulated both up and down (ANOVA; df $=2,18 ; F=7.397 ; p<0.007$ ).

targets simultaneously (SN-L7/L11; Fig. 9; $n=12$ cultures). Although some SN-L11 neuritic contacts showed very low levels of staining (one of six SN-L11 cultures and three of $12 \mathrm{SN}-\mathrm{L} 7 /$ L11 cultures), the remaining samples expressed levels that are comparable to those expressed in SN neurites contacting L7 (Figs. $8 A, 9 A)$. For example, the level of sensorin A mRNA in SN neurites contacting $\mathrm{L} 7$ was greater than that detected in neurites of the same SN contacting L11 in all cases on day 4 (seven of seven). By contrast, in $50 \%$ of the cultures on day 1 , SN neurites contacting L11 expressed sensorin A mRNA at levels that either matched or were greater than those expressed in $\mathrm{SN}$ neurites contacting L7. In the remaining three cultures, SN neurites contacting L11 expressed sensorin A transcripts at a slightly lower level than that expressed by SN neurites of the same SN contacting L7. Overall, absolute staining intensity for sensorin A fragments in SN neurites contacting L7 on day 1 was comparable to staining intensity in neurites of SNs contacting L11 (pixel intensity of $45.8 \pm 5.5$ vs $35.4 \pm 2.7$ ). Thus, modulations in the net export and targeting of sensorin A transcripts to $\mathrm{SN}$ terminals contacting appropriate postsynaptic targets develop over time in culture.

\section{DISCUSSION}

The results indicate that rRNA and selected mRNAs are exported from the cell bodies of Aplysia neurons, regenerating and forming synaptic connections in culture. Net export of a mRNA expressed only in the presynaptic $\mathrm{SN}$ is modulated by target- and branch-specific interactions.

The export of selected mRNAs into both dendritic and axonal compartments of vertebrate neurons has been observed during early stages of regeneration in cell culture (Kleiman et al., 1994; Olink and Hollenbeck, 1996; Bassell et al., 1998). Actin mRNA, a transcript synthesized in abundance by regenerating cells, is exported into the axons and regenerated neurites of SNs, L7, and L11. As is the case for mRNA encoding neuropeptides expressed in both vertebrates and invertebrates (Mohr et al., 1991; van Minnen, 1994), we also found that mRNA encoding a putative neurosecretory product that is expressed exclusively in SNs of Aplysia (Brunet et al., 1991; Santarelli et al., 1996) is expressed only in SNs and is exported to regenerated neurites and terminals of SNs. By contrast, the mRNAs encoding the isoforms of apCAM (Mayford et al., 1992) are expressed in the cell bodies of SNs and motor cells at levels comparable to those detected for actin but are not detected with our methods in axons and regenerated neurites of cells in culture for $4 \mathrm{~d}$. Sequence and structure information within individual transcripts synthesized in Aplysia neurons is likely to mediate selective export to distal processes (Wilhelm and Vale, 1993; St. Johnston, 1995; Wallace et al., 1998) via the formation of mRNA-protein complexes that interact with cytoskeletal components of the transport apparatus (Wilhelm and Vale, 1993; Knowles et al., 1996; Bassell and Singer, 1997; Oleynikov and Singer, 1998). Alternatively, many mRNAs are exported but most are degraded, whereas others are protected from degradation by their specific interactions with cytoskeletal or cytoplasmic proteins and accumulate in distal processes (Cooperstock and Lipshitz, 1997).

We also found that the level of sensorin A transcripts in the neuritic compartments of SNs contacting L7 increases with time and is highly correlated with the amplitude of the EPSP evoked in L7 on day 4. These results together with earlier findings- SN varicosities contacting L7 contain active zones for transmitter release, and the number and size of SN varicosities increase with time and correlate with EPSP amplitude (Glanzman et al., 1989, 1990; Schacher and Montarolo, 1991; Zhu et al., 1994)—suggest that exported sensorin A mRNA may accumulate at or near SN varicosities that have formed synaptic contacts with targets. The accumulation of mRNA at these sites would parallel the sites of accumulation for sensorin A neuropeptide, where expression levels of the peptide also correlate with synaptic efficacy (Santarelli et al., 1996). The correlation of sensorin A expression (mRNA and peptide) with EPSP amplitude was observed, although one of the sensorin peptides can evoke hyperpolarizations in some motor neurons (Brunet et al., 1991). The nature of the responses in L7 evoked by all peptides derived from the sensorin A precursor and the potential role of the sensorin peptides in synapse formation remain to be determined.

Is the sensorin A mRNA exported to $\mathrm{SN}$ neurites and varicosities translated, and is the translated product functional? Recent 

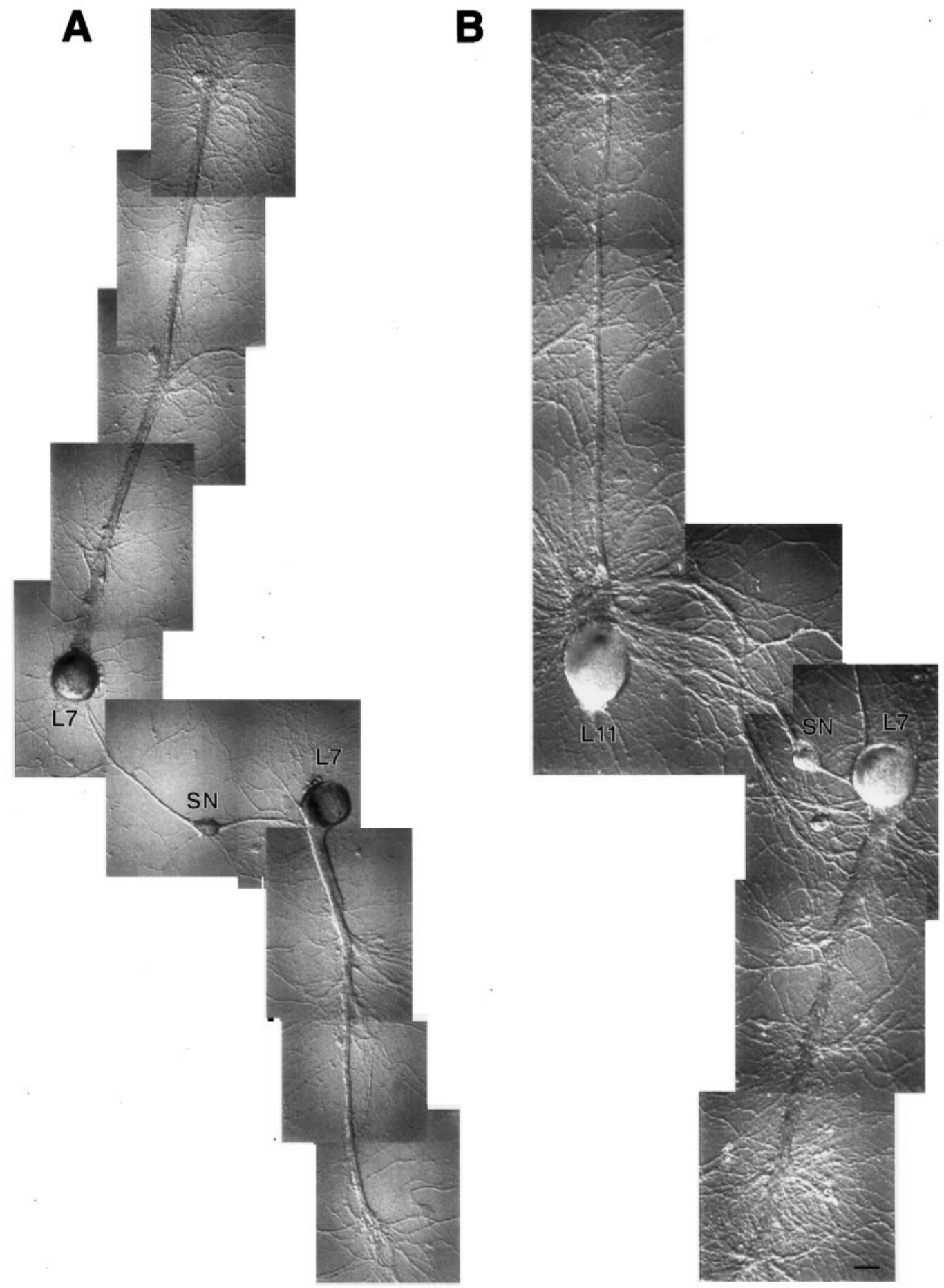

Figure 6. SN with bifurcate axon establishes separate sets of contact with two targets in culture. Shown are Nomarski contrast views of a single SN with two branches (extending left and right) contacting two targets after $4 \mathrm{~d}$ in culture. $A$, SN contacting two L7 motor cells $(S N-L 7 / L 7)$. Scale bar, $50 \mu \mathrm{m}$. $B$, SN contacting a L7 and a L11 $(S N-L 7 / L 11)$. Scale bar, $70 \mu \mathrm{m}$. Three compartments were analyzed by RT-PCR: the cell body and proximal axon of the SN ( $S N$; see compartment 1 in Fig. $1 C)$ and SN neurites from each branch interacting with the cell body, axons, and neurites of each target (compartments 2 and 3 in Fig. 1C).

studies of Martin et al. (1997) indicate that mRNA in isolated SN neurites can serve as a source for protein synthesis and that the local synthesis regulated by neuromodulators such as 5-HT can contribute to changes in synaptic efficacy and the formation of new varicosities. However, it is not known whether sensorin A neuropeptides are synthesized in isolated SN neurites or whether the locally synthesized peptides are packaged for activity- or calcium-dependent release. The packaging of local translated products into membranous organelles would require additional synthetic machinery in the distal neurites and varicosities, including the attachment of polyribosomes to endoplasmic reticulum (ER) and subsequent processing. Although ER-like structures are 

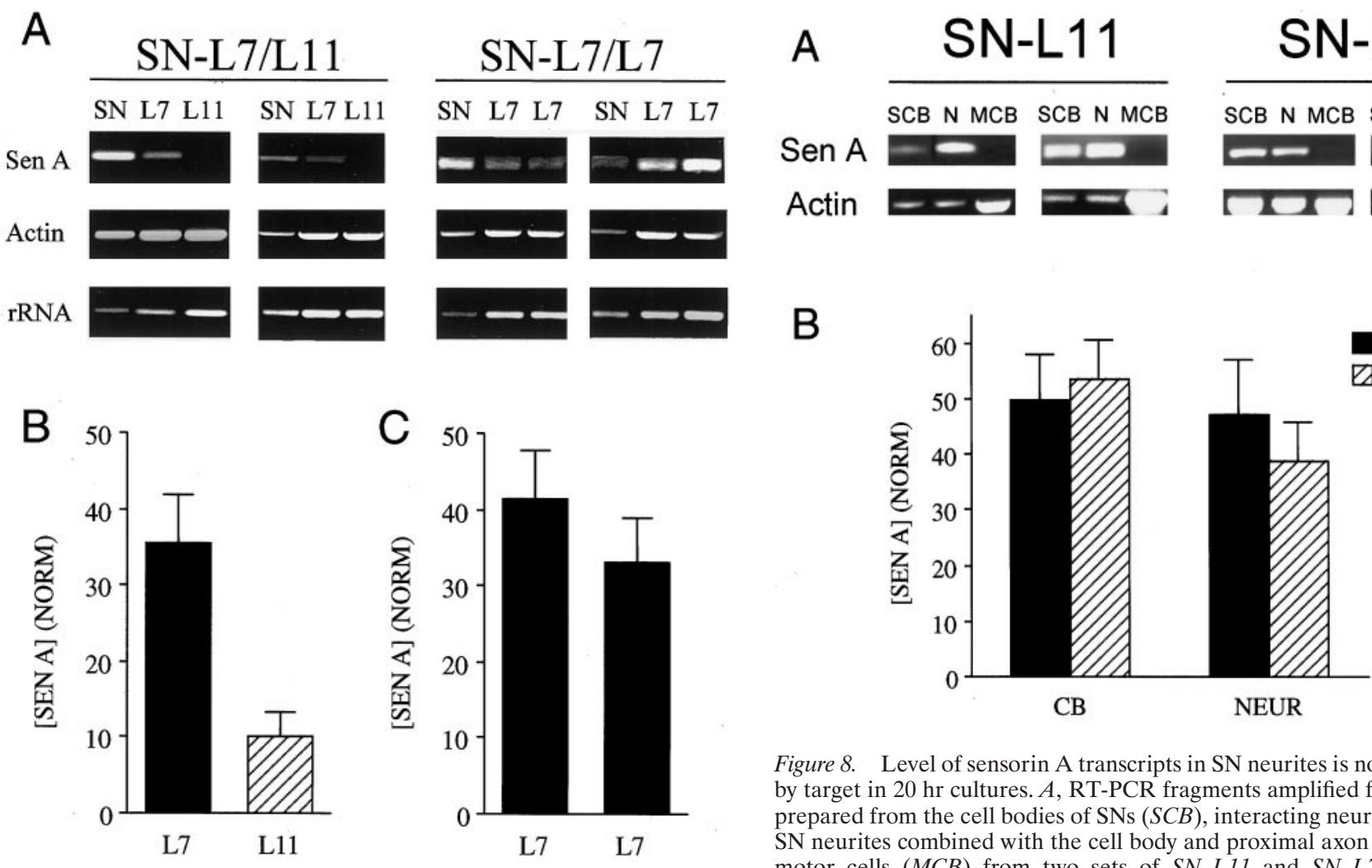

Figure 8. Level of sensorin A transcripts in SN neurites is not modulated by target in $20 \mathrm{hr}$ cultures. $A$, RT-PCR fragments amplified from samples prepared from the cell bodies of SNs $(S C B)$, interacting neurites $(N)$, and SN neurites combined with the cell body and proximal axon of the target motor cells $(M C B)$ from two sets of $S N-L 11$ and $S N-L 7$ cocultures. Fragments were synthesized with specific primers for sensorin A and actin. Unlike the situation on day 4, sensorin A was detected in the neurites of SN contacting either the neurites of L11 or L7. Little or no signal was detected in SN neurites contacting the cell bodies of L11 or L7. After $20 \mathrm{hr}$ in culture, neuritic growth from SN typically was restricted to a small region of the target as compared with overall growth by day 4 (Zhu et al., 1994; Sun and Schacher, 1996). B, Absence of modulation of levels of sensorin transcripts in SN neurites (normalized to level of actin transcripts in each compartment) at $20 \mathrm{hr}$ in culture. Two compartments were analyzed: the SN cell body $(C B)$ and neurites of SN contacting the target cell (NEUR).

L11 has varicosities and neurites for which the numbers are intermediate of those observed for SN neurites contacting L7 versus no target at all (Glanzman et al., 1989; Schacher and Montarolo, 1991). If the numbers of SN neurites and varicosities were the critical factors affecting the accumulation of sensorin A transcripts, SN neurites contacting L11 should express intermediate levels of sensorin A transcripts.

Signals from the targets appear to affect net export of sensorin A transcripts by acting locally on $\mathrm{SN}$ neurites and terminals. When a single SN contacts two L7 cells via a bifurcate axon, equivalent levels of sensorin A transcripts are detected in the terminal neurites contacting each target. By contrast, when a single SN contacts both L7 and L11, the net export to the neurites contacting L7 after $4 \mathrm{~d}$ in culture is significantly greater than the export in the other branch for which the neurites contact L11. These results, coupled with the correlation between EPSP amplitude and net export of sensorin A transcripts to SN neurites (see above), suggest that the target-derived signals from L7 or L11 can influence net accumulation and targeting of exported sensorin A mRNA at distal neurites on day 4. Moreover, they suggest that the inhibitory signals from L11 are less likely to depress initial packaging and export from the cell body. If the latter mechanism only were operative, we would predict a lower level of expression in the SN neurites contacting L7 when the other SN branch 
A

SN L7 L11 SN L7 L11 $\quad$ SN L7 L11 $\quad$ SN L7 L11

Sen A

Actin
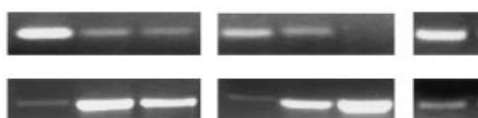

.

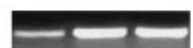

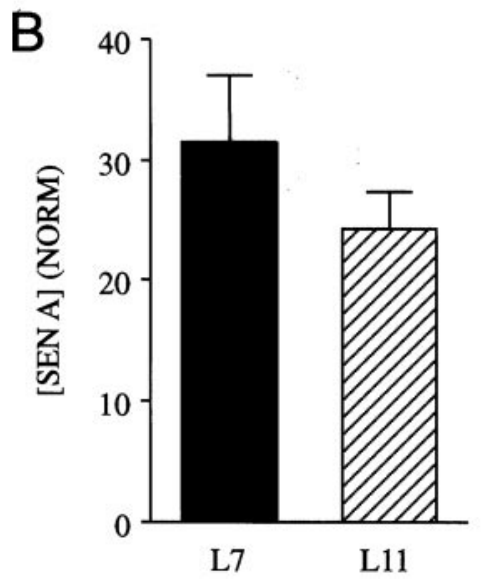

Figure 9. The target fails to regulate branch-specific accumulation of sensorin A transcripts after $20 \mathrm{hr}$ in culture. $A$, Sensorin A and actin mRNA fragments amplified from SN cell body $(S N)$ and $\mathrm{SN}$ neurites interacting with each target $(L 7$ or $L 11)$ in four sets of SN-L7/L11 cultures. In most cases (75\%) sensorin A mRNA was detected in neurites of SN contacting either L7 or L11. B, Absence of branch-specific accumulation after $20 \mathrm{hr}$. Shown are normalized levels of sensorin A mRNA in SN neurites interacting with L7 versus SN neurites of the same cell interacting with L11.

contacts an L11 as compared with expression of sensorin transcripts when each SN branch contacts a L7. This does not appear to be the case (see Fig. 7). The data, however, do not rule out the possibility that a separate signal from L7 also affects packaging and export from the SN cell body. The absolute level of sensorin A transcripts exported into neurites contacting L7 increases with time in culture. This signal, coupled with other local positive and inhibitory signals affecting transport or accumulation, can influence the net export at different branches and sites (Knowles and Kosik, 1997; Chicurel et al., 1998; Steward et al., 1998).

The target-dependent influences on net export of sensorin A transcripts develop over time. Although SNs form synapses selectively after $20 \mathrm{hr}$, we did not detect a significant difference in target- or branch-specific expression. The accumulation of sensorin A transcripts at SN branches contacting L11 at $20 \mathrm{hr}$ suggests that the local inhibitory signals from L11 induce a multi-step process in the SNs that leads over time to a reduction in net export. This scenario is reminiscent of the time course for changes observed in neuritic growth from one branch of a neuron with a bifurcate axon when the other branch contacts and forms synaptic connections with a target (Schacher, 1985). Goldberg and Schacher (1987) reported that neuritic growth at each branch is correlated with the level of axonal transport of organelles. After $1 \mathrm{~d}$ in culture and the early establishment of synaptic contact at one branch, the growth and axonal transport at each branch is equivalent. With time, there is a downregulation of growth and organelle transport (both orthograde and retrograde) in the branch without the target, whereas the branch contacting the target continues to grow, form contacts with the target, and has normal levels of organelle transport. Thus, the presence of a target at one branch leads over time to selective stabilization of the transport apparatus in one branch and destabilization of the transport apparatus in the other branch. In the experiments reported here, the retrograde signals from L11 and L7 may initiate local alterations in the cytoskeletal or transport machinery of the branches and neurites, thereby modulating net export of sensorin A mRNA to SN neurites contacting L11 versus L7.

One model explaining our results is that different targetderived signals affect the organization of the cytoplasmic apparatus responsible for the export and accumulation of stable transcripts at developing and maturing synaptic contacts. The signals can influence export at three sites (Steward et al., 1998). First, signals from L7 may influence the initial packaging of the mRNAs for export from the cell body by modulating the expression of binding proteins, cytoskeletal elements, and transport motors necessary for the export of the mRNA-protein complexes. Second, signals from L7 or L11 may stabilize or destabilize, respectively, the transport apparatus within the axon allowing export of mRNA-protein complexes to developing or maturing synapses. Third, signals from the targets modulate the organization of synaptic contacts that may be required for the local accumulation of stable mRNAs. This could be accomplished by appropriate expression of specific components of the cytoskeleton within varicosities that bind the mRNA, thereby preventing degradation by cytoplasmic RNases and facilitating translation. Future experiments will examine the nature of the target-derived signals, their effect on each step in the export and accumulation of mRNAs, and the contribution and function of local translated products to synapse formation and maturation.

\section{REFERENCES}

Ambron RT, Den H, Schacher S (1985) Synaptogenesis by single identified neurons in vitro: contribution of rapidly transported and newly synthesized proteins. J Neurosci 5:2857-2865.

Bailey CH, Thompson EB, Castellucci VF, Kandel ER (1979) Ultrastructure of the synapses of sensory neurons that mediate the gill withdrawal reflex in Aplysia. J Neurocytol 8:415-444.

Bassell GJ, Singer RH (1997) mRNA and cytoskeletal filaments. Curr Opin Cell Biol 9:109-115.

Bassell GJ, Zhang H, Byrd AL, Femino AM, Singer RH, Taneja KL, Lifshitz LM, Herman IM, Kosik KS (1998) Sorting of $\beta$-actin mRNA and protein to neurites and growth cones in culture. J Neurosci 18:251-265.

Brunet J-F, Shapiro E, Foster SA, Kandel ER, Yuichi I (1991) Identification of a peptide specific for Aplysia sensory neurons by PCR-based differential screening. Science 252:856-859.

Cabelli RJ, Shelton DL, Segal RA, Shatz CJ (1997) Blockade of endogenous ligands of trkB inhibits formation of ocular dominance columns. Neuron 19:63-76.

Camardo J, Proshansky E, Schacher S (1983) Identified Aplysia neurons form specific chemical synapses in culture. J Neurosci 3:2614-2620.

Casadio A, Martin KC, Bailey CH, Chen M, Zhu H, Kandel ER (1997) Molecular mechanisms underlying synapse-specific long-term facilitation of Aplysia sensory to motor synapses. Soc Neurosci Abstr 24:234.

Chicurel ME, Singer RH, Meyer CJ, Ingber DE (1998) Integrin binding and mechanical tension induce movement of mRNA and ribosomes to focal adhesions. Nature 392:730-733.

Cooperstock RL, Lipshitz HD (1997) Control of mRNA stability and translation during Drosophila development. Semin Cell Dev Biol 8:541-549.

Crino PB, Eberwine J (1996) Molecular characterization of the dendritic growth cone: regulated mRNA transport and local protein synthesis. Neuron 17:1173-1187.

DesGroseillers L, Auclair D, Wickham L, Maalouf M (1994) A novel actin cDNA is expressed in the neurons of Aplysia californica. Biochim Biophys Acta 121:322-324. 
Fitzsimonds RM, Poo M-M (1998) Retrograde signaling in the development and modification of synapses. Physiol Rev 78:143-170.

Glanzman DL, Kandel ER, Schacher S (1989) Identified target motor neuron regulates neurite outgrowth and synapse formation of Aplysia sensory neurons in vitro. Neuron 3:441-450.

Glanzman DL, Kandel ER, Schacher S (1990) Target-dependent structural changes accompanying long-term synaptic facilitation in Aplysia neurons. Science 249:799-802.

Goldberg DJ, Schacher S (1987) Differential growth of the two branches of a regenerating bifurcate axon is associated with differential axonal transport of organelles. Dev Biol 124:35-40.

Hawver D, Schacher S (1993) Selective fasciculation as a mechanism for the formation of specific chemical connections between Aplysia neurons in vitro. J Neurobiol 24:368-383.

Keller F, Schacher S (1990) Neuron-specific membrane glycoproteins promoting neurite fasciculation in Aplysia californica. J Cell Biol 111:2637-2650.

Knowles RB, Kosik KS (1997) Neurotrophin-3 signals redistribute RNA in neurons. Proc Natl Acad Sci USA 94:14804-14808.

Knowles RB, Sabry JH, Martone ME, Deerinck TJ, Ellisman MH, Bassell GJ, Kosik KS (1996) Translocation of RNA granules in living neurons. J Neurosci 16:7812-7820.

Kleiman R, Banker G, Steward O (1994) Development of subcellular mRNA compartmentation in hippocampal neurons in culture. J Neurosci 14:1130-1140.

Martin KC, Casadio A, Zhu H, Yaping E, Rose JC, Chen M, Bailey CH, Kandel ER (1997) Synapse-specific, long-term facilitation of Aplysia sensory to motor synapses: a function for local protein synthesis in memory storage. Cell 91:927-938.

Mayford M, Barzilai A, Keller F, Schacher S, Kandel ER (1992) Modulation of an NCAM-related adhesion molecule with long-term synaptic plasticity in Aplysia. Science 256:638-644.

Mohr E, Fehr S, Richter D (1991) Axonal transport of neuropeptide encoding mRNAs within the hypothalamo-hypophaseal tract of rats. EMBO J 10:2419-2424.

Montarolo PG, Goelet P, Castellucci VF, Morgan J, Kandel ER, Schacher S (1986) A critical period for macromolecular synthesis in long-term heterosynaptic facilitation in Aplysia. Science 234:1249-1254.

Oleynikov Y, Singer RH (1998) RNA localization: different zip codes, same postman? Trends Cell Biol 8:381-383.

Olink-Coux M, Hollenbeck PJ (1996) Localization and active transport of mRNA in axons of sympathetic neurons in culture. J Neurosci 16:1346-1358.

Rayport SG, Schacher S (1986) Synaptic plasticity in vitro: cell culture of identified Aplysia neurons mediating short-term habituation and sensitization. J Neurosci 6:759-763.

Sanes JR, Apel ED, Gautam M, Glass D, Grady RM, Martin PT, Nicholl MC, Yancopoulus GD (1998) Agrin receptors at the skeletal neuromuscular junction. Ann NY Acad Sci 841:1-13.
Santarelli L, Montarolo PG, Schacher S (1996) Neuropeptide localization in varicosities of Aplysia sensory neurons is regulated by target and neuromodulators evoking long-term synaptic plasticity. J Neurobiol 31:297-308.

Schacher S (1985) Differential synapse formation and neurite outgrowth at two branches of the metacerebral cell of Aplysia in dissociated cell culture. J Neurosci 5:2028-2034.

Schacher S, Montarolo PG (1991) Target-dependent structural changes in sensory neurons of Aplysia accompany long-term heterosynaptic inhibition. Neuron 6:679-690.

Schacher S, Proshansky E (1983) Neurite regeneration by Aplysia neurons in dissociated cell culture: modulation by Aplysia hemolymph and the presence of the initial axon segment. J Neurosci 3:2403-2413.

Steward O (1997) mRNA localization in neurons: a multipurpose mechanism? Neuron 18:9-12.

Steward O, Wallace CS, Lyford GL, Worley PF (1998) Synaptic activation causes the mRNA for the IEG Arc to localize selectively near activated postsynaptic sites on dendrites. Neuron 21:741-751.

St. Johnston D (1995) The intracellular localization of mRNAs. Cell 81:161-170.

Sun Z-Y, Schacher S (1996) Development of short-term heterosynaptic facilitation at Aplysia sensorimotor synapses in vitro is accompanied by changes in the functional expression of presynaptic serotonin receptors. J Neurophysiol 76:2250-2261.

van Minnen J (1994) Axonal localization of neuropeptide-encoding mRNA in identified neurons of the snail Lymnaea stagnalis. Cell Tissue Res 276:155-161.

van Minnen J, Bergman JJ, Van Kesteren ER, Smit AB, Geraerts WPM, Lukowiak K, Hasan SU, Syed NI (1997) De novo protein synthesis in isolated axons of identified neurons. Neuroscience 80:1-7.

Wallace CS, Lyford GL, Worley PF, Steward O (1998) Differential intracellular sorting of immediate early gene mRNAs depends on signals in the mRNA sequence. J Neurosci 18:26-35.

Wilhelm JE, Vale RD (1993) RNA on the move: the mRNA localization pathway. J Cell Biol 123:269-274.

Winberg ML, Mitchell KJ, Goodman CS (1998) Genetic analysis of the mechanisms controlling target selection: complementary and combinatorial functions of netrins, semaphorins, and IgCams. Cell 93:581-592.

Winnepenninckx B, Steiner G, Backeljau T, De Wachter R (1998) Details of gastropod phylogeny inferred from $18 \mathrm{~S}$ rRNA sequences. Mol Phylogenet Evol 9:55-63.

Wu L, Wells D, Tay J, Mendis D, Abbott MA, Barnitt A, Quinlan E, Heynen A, Fallon JR, Richter JD (1998) CPEB-mediated cytoplasmic polyadenylation and the regulation of experience-dependent translation of $\alpha$-CaMKII mRNA at synapses. Neuron 21:1129-1139.

Zhu H, Wu F, Schacher S (1994) Aplysia cell adhesion molecules and serotonin regulate sensory cell-motor cell interactions during early stages of synapse formation in vitro. J Neurosci 14:6886-6900. 